\title{
The Ulam Type Stability of a Generalized Additive Mapping and Concrete Examples
}

\author{
Hiroyoshi Oda, ${ }^{1}$ Makoto Tsukada, ${ }^{1}$ Takeshi Miura, ${ }^{2}$ Yuji Kobayashi, ${ }^{3}$ and Sin-Ei Takahasi ${ }^{4}$ \\ ${ }^{1}$ Department of Information Sciences, Toho University, Funabashi, Chiba 274-8501, Japan \\ ${ }^{2}$ Yamagata University, Yonezawa, Yamagata 273-0866, Japan \\ ${ }^{3}$ Toho University, Funabashi, Chiba 274-8501, Japan \\ ${ }^{4}$ Toho University, Yamagata University, Funabashi, Chiba 273-0866, Japan
}

Correspondence should be addressed to Makoto Tsukada; tsukada@is.sci.toho-u.ac.jp

Received 27 December 2012; Accepted 21 February 2013

Academic Editor: Irena Lasiecka

Copyright (C) 2013 Hiroyoshi Oda et al. This is an open access article distributed under the Creative Commons Attribution License, which permits unrestricted use, distribution, and reproduction in any medium, provided the original work is properly cited.

We give an Ulam type stability result for the following functional equation: $f\left(\alpha x-\alpha x^{\prime}+x_{0}\right)=\beta f(x)-\beta f\left(x^{\prime}\right)+y_{0}$ (for all $\left.x, x^{\prime} \in X\right)$ under a suitable condition. We also give a concrete stability result for the case taking up $\delta\|x\|^{p}\left\|x^{\prime}\right\|^{q}$ as a control function.

\section{Introduction}

In 1940, Ulam [1] proposed the following stability problem: "When is it true that a function which satisfies some functional equation approximately must be close to one satisfying the equation exactly?" Next year, Hyers [2] gave an answer to this problem for additive mappings between Banach spaces. Furthermore, Aoki [3] and Rassias [4] obtained independently generalized results of Hyers' theorem which allow the Cauchy difference to be unbounded.

Let $X$ and $Y$ be normed spaces over $\mathbb{K}$, which denotes either the real field $\mathbb{R}$ or the complex field $\mathbb{C}$. Throughout the paper, we fix scalars $a, b, c, d \in \mathbb{K} \backslash\{0\}$ and vectors $x_{0} \in X$ and $y_{0} \in Y$. We say that a mapping $f$ of $X$ into $Y$ is $\left(a, b, c, d ; x_{0}, y_{0}\right)$-additive if

$$
f\left(a x+b x^{\prime}+x_{0}\right)=c f(x)+d f\left(x^{\prime}\right)+y_{0}
$$

for all $x, x^{\prime} \in X$. When $x_{0}=y_{0}=0$, we say it to be $(a, b, c, d)$-additive. Aczél [5] specified what this generalized Cauchy equation is. The Ulam type stability problem for such an $f$ has been investigated in [6-8]. However, these results have been obtained in cases where either $a+b \neq 0$ or $c+d \neq 0$ (see Theorems $\mathrm{A}$ and $\mathrm{B}$ ). In this paper, we will investigate the problem for $\left(a,-a, c,-c ; x_{0}, y_{0}\right)$-additive mappings, that is, in the case $a+b=c+d=0$. In Section 2, we state the details of $\left(a,-a, c,-c ; x_{0}, y_{0}\right)$-additive mappings (Theorem 3). In Section 3, we give our main results about the stability for them (see Theorems 7-10). In the final section, we apply the results to some concrete examples, where we take up $\delta\|x\|^{p}\left\|x^{\prime}\right\|^{q}$ as a control function $\varepsilon\left(x, x^{\prime}\right)$ (see Corollaries 11-14).

\section{2. $\left(a,-a, c,-c ; x_{0}, y_{0}\right)$-Additive Mappings}

The following result asserts that any $\left(a,-a, c,-c ; x_{0}, y_{0}\right)$ additive mapping is transformed into some $(a,-a, c,-c)$ additive mapping by a certain translation and that any $(a,-a, c,-c)$-additive mapping is an additive mapping in usual sense with some extra condition.

Proposition 1. Let $f$ and $g$ be two mappings of $X$ into $Y$ such that $g(x)=f\left(x+x_{0}\right)-y_{0}$ for all $x \in X$. Then the following three statements are equivalent:

(i) $f$ is $\left(a,-a, c,-c ; x_{0}, y_{0}\right)$-additive,

(ii) $g$ is $(a,-a, c,-c)$-additive,

(iii) $g$ is additive and $g(a x)=c g(x)$ for all $x \in X$. 
Proof. (i) $\Leftrightarrow$ (ii) Since

$$
\begin{aligned}
f(a x & \left.-a x^{\prime}+x_{0}\right) \\
& =g\left(a x-a x^{\prime}\right)+y_{0} \\
& =g\left(a\left(x-x_{0}\right)-a\left(x^{\prime}-x_{0}\right)\right)+y_{0}, \\
c(x) & -c f\left(x^{\prime}\right)+y_{0} \\
& =c\left(f(x)-y_{0}\right)-c\left(f\left(x^{\prime}\right)-y_{0}\right)+y_{0} \\
& =c g\left(x-x_{0}\right)-c g\left(x^{\prime}-x_{0}\right)+y_{0}
\end{aligned}
$$

for all $x, x^{\prime} \in X$, it follows that

$$
\begin{aligned}
& f:\left(a,-a, c,-c ; x_{0}, y_{0}\right) \text {-additive } \\
& \Leftrightarrow g\left(a\left(x-x_{0}\right)-a\left(x^{\prime}-x_{0}\right)\right)+y_{0}=c g\left(x-x_{0}\right)-c g\left(x^{\prime}-\right. \\
& \left.x_{0}\right)+y_{0}\left(\text { for all } x, x^{\prime} \in X\right) \\
& \Leftrightarrow g\left(a x-a x^{\prime}\right)=c g(x)-c g\left(x^{\prime}\right)\left(\text { for all } x, x^{\prime} \in X\right) \\
& \Leftrightarrow g:(a,-a, c,-c) \text {-additive. }
\end{aligned}
$$

(ii) $\Rightarrow$ (iii) Suppose that

$$
g\left(a x-a x^{\prime}\right)=c g(x)-c g\left(x^{\prime}\right)
$$

for all $x, x^{\prime} \in X$. When $x=x^{\prime}$, we have $g(0)=0$. Using this, $g(a x)=c g(x)$ and also $g(-a x)=-c g(x)$ for all $x \in X$. Therefore,

$$
\begin{aligned}
g\left(x+x^{\prime}\right) & =g\left(a \frac{x}{a}-a\left(-\frac{x^{\prime}}{a}\right)\right) \\
& =c g\left(\frac{x}{a}\right)-c g\left(-\frac{x^{\prime}}{a}\right) \\
& =c g\left(\frac{x}{a}\right)+(-c) g\left(-\frac{x^{\prime}}{a}\right)=g(x)+g\left(x^{\prime}\right)
\end{aligned}
$$

for all $x, x^{\prime} \in X$.

(iii) $\Rightarrow$ (ii) Because $g(-x)=-g(x)$ for all $x \in X$ (see also the following remark), it is trivial.

Remark 2. We denote by $\mathbb{Q}$ the field of all rational numbers. It is well known that if $g$ is additive, then $g(q x)=q g(x)$ for every $q \in \mathbb{Q}$ and $x \in X$, that is, $g$ is $\mathbb{Q}$-linear. Hence, if $g$ is additive and continuous, $g$ must be $\mathbb{R}$-linear. On the other hand, when $\mathbb{K}=\mathbb{C}$, we have a lot of continuous additive nonlinear mappings by considering the composition of linear transformations on $\mathbb{R}^{2}$ and the $\mathbb{R}$-linear isometry $(x, y) \mapsto$ $x+i y$.

The constant $y_{0}$ is a trivial $\left(a,-a, c,-c ; x_{0}, y_{0}\right)$-additive mapping of $X$ into $Y$. The following theorem says that unless it is a unique $\left(a,-a, c,-c ; x_{0}, y_{0}\right)$-additive mapping, discontinuous one always exists.

Theorem 3. (I) If $a=c$, then there exists a discontinuous $\left(a,-a, c,-c ; x_{0}, y_{0}\right)$-additive mapping of $X$ into $Y$.

(II) If $a \neq c$, then the followings hold: (i) If both $a$ and $c$ are transcendental numbers, then there exists a discontinuous $\left(a,-a, c,-c ; x_{0}, y_{0}\right)$-additive mapping of $X$ into $Y$.

(ii) If one of $a$ and $c$ is transcendental and the other is algebraic, then the constant $y_{0}$ is a unique $(a,-a, c$, $\left.-c ; x_{0}, y_{0}\right)$-additive mapping of $X$ into $Y$.

(iii) If both $a$ and $c$ are algebraic with a common minimal polynomial, then there exists a discontinuous ( $a,-a$, $\left.c,-c ; x_{0}, y_{0}\right)$-additive mapping of $X$ into $Y$.

(iv) If $a$ and $c$ are algebraic with distinct minimal polynomials, then the constant $y_{0}$ is a unique $(a,-a, c$, $\left.-c ; x_{0}, y_{0}\right)$-additive mapping of $X$ into $Y$.

Moreover, when $\mathbb{K}=\mathbb{R}$, there is no nontrivial continuous $\left(a,-a, c,-c ; x_{0}, y_{0}\right)$-additive mapping $f$ of $X$ into $Y$. On the other hand, when $\mathbb{K}=\mathbb{C}$, if $a$ is not real and $a=\bar{c}$ (the complex conjugate of $c)$, then the mapping $x \mapsto f\left(x+x_{0}\right)-$ $y_{0}$ must be conjugate linear for every continuous $(a,-a, c$, $\left.-c ; x_{0}, y_{0}\right)$-additive mapping $f$ of $X$ into $Y$.

In order to show Theorem 3, we need some lemmas for $\mathbb{K}$-valued $(a,-a, c,-c)$-additive functions defined on $\mathbb{K}$. For any $x \in \mathbb{K}$, we denote by $\mathbb{Q}(x)$ the subfield of $\mathbb{K}$ generated by $x$ over $\mathbb{Q}$.

By the following proofs of Lemma 6 and Theorem 3, if there is a discontinuous $\left(a,-a, c,-c ; x_{0}, y_{0}\right)$-additive mapping, then there are sufficiently many such mappings in the sense that there exists such a mapping which separates any $\mathbb{Q}(a)$-linear independent points of $X$.

Lemma 4. Any $(a,-a, c,-c)$-additive $\varphi$ of $\mathbb{K}$ into itself satisfies $\varphi(p(a) x)=p(c) \varphi(x)$ for all $x \in \mathbb{K}$ and $p(X) \in \mathbb{Q}[X]$.

Proof. Note that $\varphi$ is additive and $\varphi(a x)=c \varphi(x)$ for each $x \in \mathbb{K}$ by Proposition 1 . Let $p(X)=a_{0}+a_{1} X+\cdots+a_{n} X^{n}$ with $a_{0}, a_{1}, \ldots, a_{n} \in \mathbb{Q}$. Since $\varphi$ is $\mathbb{Q}$-linear,

$$
\begin{aligned}
\varphi(p(a) x) & =a_{0} \varphi(x)+a_{1} \varphi(a x)+\cdots+a_{n} \varphi\left(a^{n} x\right) \\
& =a_{0} \varphi(x)+a_{1} c \varphi(x)+\cdots+a_{n} c^{n} \varphi(x) \\
& =p(c) \varphi(x)
\end{aligned}
$$

for all $x \in \mathbb{K}$.

Lemma 5. Let $E$ and $F$ be subfields of $\mathbb{K}$ and $\psi$ an isomorphism of $E$ onto $F$. Then, $\psi$ has an additive bijective extension $\varphi$ to the full space $\mathbb{K}$ such that

$$
\varphi(\varepsilon x)=\psi(\varepsilon) \varphi(x)
$$

for all $\varepsilon \in E$ and $x \in \mathbb{K}$. Moreover, one has a discontinuous one whenever $\mathbb{R} \backslash E \neq \emptyset$.

Proof. Let $\left\{e_{j}: j \in J\right\}$ and $\left\{f_{j}: j \in J^{\prime}\right\}$ be an $E$-linear base and an $F$-linear base of $\mathbb{K}$, respectively. Because both of them have same cardinality, we take $J=J^{\prime}$. Moreover, we may assume without loss of generality that $e_{j_{0}}=f_{j_{0}}=1$ for some $j_{0} \in J$. 
For any $x \in \mathbb{K}$, there exist a finite $I \subset J$ and $\left\{x_{i}\right\}_{i \in I} \subseteq E$ such that

$$
x=\sum_{i \in I} x_{i} e_{i}
$$

and this decomposition is unique. Hence, we can define $\varphi$ : $\mathbb{K} \rightarrow \mathbb{K}$ by

$$
\varphi(x)=\sum_{i \in I} \psi\left(x_{i}\right) f_{i}
$$

This $\varphi$ is a desired extension.

In order to get a discontinuous extension, we consider the base $\left\{e_{j}: j \in J\right\}$ and $\left\{f_{j}: j \in J\right\}$ such that $f_{j_{1}} \neq e_{j_{1}} \in \mathbb{R} \backslash E$ for some $j_{1} \in J$. Because $\mathbb{Q} \subseteq E$, take a rational sequence $\left\{\varepsilon_{n}\right\}$ converging to $e_{j_{1}}$. Suppose that $\varphi$ is continuous. Then,

$$
\begin{gathered}
\varphi\left(\varepsilon_{n}\right) \longrightarrow \varphi\left(e_{j_{1}}\right)=f_{j_{1}}, \\
\varphi\left(\varepsilon_{n}\right)=\psi\left(\varepsilon_{n}\right)=\varepsilon_{n} \longrightarrow e_{j_{1}},
\end{gathered}
$$

as $n \rightarrow \infty$. This is contradiction. Thus $\varphi$ is discontinuous.

\section{Lemma 6. Suppose that $a \neq c$.}

(i) If both $a$ and $c$ are transcendental numbers, then there exists a discontinuous $(a,-a, c,-c)$-additive function of $\mathbb{K}$ into itself.

(ii) If one of $a$ and $c$ is transcendental and the other is algebraic, then the constant 0 is a unique $(a,-a, c,-c)$ additive function of $\mathbb{K}$ into itself.

(iii) If both $a$ and $c$ are algebraic with a common minimal polynomial, then there exists a discontinuous $(a,-a, c,-c)$-additive function of $\mathbb{K}$ into itself.

(iv) If $a$ and $c$ are algebraic with distinct minimal polynomials, then the constant 0 is a unique $(a,-a, c,-c)$ additive function of $\mathbb{K}$ into itself.

Moreover, when $\mathbb{K}=\mathbb{R}$, every nontrivial $(a,-a, c,-c)$-additive function of $\mathbb{K}$ into itself is discontinuous. On the other hand, when $\mathbb{K}=\mathbb{C}$, if $a$ is not real and $a=\bar{c}$, then any continuous $(a,-a, c,-c)$-additive function $f$ of $\mathbb{C}$ into itselfmust be ofform $f(x)=\alpha \bar{x}(x \in \mathbb{C})$ for some $\alpha \in \mathbb{C}$.

Proof. (i) Suppose that both $a$ and $c$ are transcendental. Then, $\mathbb{Q}(a)$ (resp., $\mathbb{Q}(c)$ ) is isomorphic to the rational function field in indeterminate $a$ (resp., $c$ ). So, the substitution $a \rightarrow c$ induces an isomorphism $\varphi_{a c}: \mathbb{Q}(a) \rightarrow \mathbb{Q}(c)$ of fields. By Lemma 5 , because $\mathbb{R} \backslash \mathbb{Q}(a) \neq \emptyset, \varphi_{a c}$ has a discontinuous additive extension $\varphi$ to $\mathbb{K}$ such that $\varphi(\varepsilon x)=\varphi_{a c}(\varepsilon) \varphi(x)$ for every $\varepsilon \in \mathbb{Q}(a)$ and $x \in \mathbb{K}$. Then, $\varphi(a x)=\varphi_{a c}(a) \varphi(x)=$ $c \varphi(x)$ for all $x \in \mathbb{K}$, and, hence, $\varphi$ is $(a,-a, c,-c)$-additive by Proposition 1.

(ii) Let $\varphi$ be any $(a,-a, c,-c)$-additive function. If $a$ is transcendental and $c$ is algebraic with nonzero polynomial such that $p(c)=0$, then from Lemma 4 , we have

$$
\varphi(x)=\varphi\left(p(a) p(a)^{-1} x\right)=p(c) \varphi\left(p(a)^{-1} x\right)=0
$$

for all $x \in \mathbb{K}$. If $c$ is transcendental and $a$ is algebraic with nonzero polynomial such that $q(a)=0$, then from Lemma 4 , we have

$$
\varphi(x)=\frac{1}{q(c)} q(c) \varphi(x)=\frac{1}{q(c)} \varphi(q(a) x)=\frac{1}{q(c)} \varphi(0)=0
$$

for all $x \in \mathbb{K}$.

(iii) If $a$ is algebraic with minimal polynomial $p(X) \epsilon$ $\mathbb{Q}[X]$, then $\mathbb{Q}(a)$ consists of all polynomials $f(a)$ in $a$ of degree up to deg $p-1$. So, if $c$ is also algebraic with the same minimal polynomial $p$, then the substitution $a \rightarrow c$ induces an automorphism $\varphi_{a c}: \mathbb{Q}(a) \rightarrow \mathbb{Q}(c)=\mathbb{Q}(a)$. As same as (i), $\varphi_{a c}$ has a discontinuous $(a,-a, c,-c)$-additive extension.

(iv) Suppose that $a$ and $c$ are algebraic with distinct minimal polynomials $p$ and $q$ over the field $\mathbb{Q}$, respectively. Let $\varphi$ be any $(a,-a, c,-c)$-additive function. To show $\varphi=$ 0 , we assume, on the contrary, that there is an $x_{0} \in \mathbb{K}$ with $\varphi\left(x_{0}\right) \neq 0$. Then, from Lemma 5 , we have $p(c) \varphi\left(x_{0}\right)=$ $\varphi\left(p(a) x_{0}\right)=\varphi(0)=0$, and hence $p(c)=0$. This contradicts the prerequisite for $a$ and $c$. Hence, $\varphi$ must be zero.

When $\mathbb{K}=\mathbb{R}$, since every continuous additive function is $\mathbb{R}$-linear and $a \neq c$, there is no continuous $(a,-a, c,-c)$ additive function by Proposition 1 . Now, we consider the case $\mathbb{K}=\mathbb{C}$. Let $\varphi$ be a nontrivial continuous $(a,-a, c,-c)$-additive function. Note that $\varphi$ is $\mathbb{R}$-linear. If $a$ is not real and $a=\bar{c}$, we can easily see that $\varphi(i x)=-i \varphi(x)$ for all $x \in \mathbb{C}$. Thus, $\varphi$ is conjugate linear, and hence $\varphi(x)=\alpha \bar{x}(x \in \mathbb{C})$, where $\alpha=\varphi(1)$.

Proof of Theorem 3. (I) We assume without loss of generality that $x_{0}=y_{0}=0$ with the help of Proposition 1. Given an $(a,-a, c,-c)$-additive function $\varphi$, take a $y_{1} \in Y$ with $\left\|y_{1}\right\|=1$ and a nonzero functional $h$ in $X^{*}$, the dual space of $X$. Put

$$
f(x)=\varphi(h(x)) y_{1} \quad(x \in X) .
$$

Then, we can easily see that $f$ is an $(a,-a, c,-c)$-additive mapping of $X$ into $Y$. Also, if $\varphi$ is discontinuous, then so is $f$. In fact, if $\varphi$ is discontinuous, we can find a sequence $\left\{a_{n}\right\}$ in $\mathbb{K}$ such that $\lim _{n \rightarrow \infty} a_{n}=0$ and $\left|\varphi\left(a_{n}\right)\right| \geq 1(n=1,2, \ldots)$. Choose an $x_{1}$ in $X$ with $h\left(x_{1}\right)=1$ and put $x_{n}=a_{n} x_{1}$ for each $n \in \mathbb{N}$. Then, $\left\|x_{n}\right\|=\left|a_{n}\right|\left\|x_{1}\right\| \rightarrow 0$ as $n \rightarrow \infty$ and $\left\|f\left(x_{n}\right)\right\|=\left|\varphi\left(a_{n}\right)\right| \geq 1(n=1,2, \ldots)$, so $f$ is discontinuous, as required. Therefore Theorem 3(I), (II)-(i), and (II)-(iii) follow easily from Lemmas 4 and 6 .

Given an $(a,-a, c,-c)$-additive mapping $f$ of $X$ into $Y$, take $x \in X$ and $h \in Y^{*}$ arbitrarily, and put $\varphi(t)=h(f(t x))$ for each $t \in \mathbb{K}$. Then, $\varphi: \mathbb{K} \rightarrow \mathbb{K}$ is $(a,-a, c,-c)$-additive. If $\varphi=0$ for each $h \in Y^{*}$ and $x \in X$, then $f=0$ by the HahnBanach theorem. Therefore, Theorem 3(II)-(ii) and (II)-(iv) follow easily from Lemma 6. The final assertion in (II) also follows from Lemma 6 and its proof.

\section{A Stability of Generalized Additive Mappings}

In this section, we consider a couple of cases which are left out in [8] about the Ulam type stability. We take a nonnegative 
function $\varepsilon$ (say a control function) on $X \times X$ and also a certain nonnegative function $\delta$ on $X$ which depends on $\varepsilon$. We say that a system of all $\left(a, b, c, d ; x_{0}, y_{0}\right)$-additive mappings is strictly $(\varepsilon, \delta)$-stable whenever the following statement is true:

"If a mapping $f$ of $X$ into $Y$ satisfies

$$
\left\|f\left(a x+b x^{\prime}+x_{0}\right)-c f(x)-d f\left(x^{\prime}\right)-y_{0}\right\| \leq \varepsilon\left(x, x^{\prime}\right)
$$

for all $x, x^{\prime} \in X$, then there exists a unique $\left(a, b, c, d ; x_{0}, y_{0}\right)$ additive mapping $f_{\infty}$ such that

$$
\left\|f(x)-f_{\infty}(x)\right\| \leq \delta(x)
$$

for all $x \in X$."

Throughout the remainder of this paper, we assume that $Y$ is a Banach space. This is because all of our results depend on the following theorems whose proofs need the Banach fixed point theorem.

Theorem A (see [8, Theorem 3.1]). Let $a+b \neq 0$ and $K \geq 0$ with $K|c+d|<1$. One takes a control function $\varepsilon$ which satisfies

$$
\varepsilon\left(x, x^{\prime}\right) \leq K \varepsilon\left((a+b) x+x_{0},(a+b) x^{\prime}+x_{0}\right)
$$

for all $x, x^{\prime} \in X$ and puts

$$
\delta(x)=\frac{K \varepsilon(x, x)}{1-K|c+d|}
$$

for each $x \in X$.

Then, the strict $(\varepsilon, \delta)$-stability holds for the system of $(a, b$, $\left.c, d ; x_{0}, y_{0}\right)$-additive mappings.

Theorem B (see [8, Theorem 3.2]). Let $c+d \neq 0$ and $K \geq 0$ with $K<|c+d|$. One takes a control function $\varepsilon$ which satisfies

$$
\varepsilon\left((a+b) x+x_{0},(a+b) x^{\prime}+x_{0}\right) \leq K \varepsilon\left(x, x^{\prime}\right)
$$

for all $x, x^{\prime} \in X$ and puts

$$
\delta(x)=\frac{\varepsilon(x, x)}{|c+d|-K}
$$

for each $x \in X$.

Then, the strict $(\varepsilon, \delta)$-stability holds for the system of $\left(a, b, c, d ; x_{0}, y_{0}\right)$-additive mappings.

Both of these theorems do not say about $(a,-a, c$, $\left.-c ; x_{0}, y_{0}\right)$-additive mappings at all; however, we will get the following stability theorems for them. Theorem 7 is of the case $a \neq-1$, Theorem 8 is of the case $b \neq-1$, and Theorems 9 and 10 are for $\left(-1,1,-1,1 ; x_{0}, y_{0}\right)$-additive mappings. These cover all of the systems of $\left(a,-a, c,-c ; x_{0}, y_{0}\right)$-additive mappings.

Theorem 7. Let $a+1 \neq 0$ and $K \geq 0$ with $K|1+c|<|c|$. One takes a control function $\varepsilon$ which satisfies

$$
\varepsilon\left(x, x^{\prime}\right) \leq K \varepsilon\left(\left(a^{-1}+1\right) x-a^{-1} x_{0},\left(a^{-1}+1\right) x^{\prime}-a^{-1} x_{0}\right)
$$

for all $x, x^{\prime} \in X$, and puts

$$
\delta(x)=\frac{K \varepsilon\left(\left(a^{-1}+1\right) x-a^{-1} x_{0}, x\right)}{|c|-K|1+c|}
$$

for each $x \in X$.

Then, the strict $(\varepsilon, \delta)$-stability holds for the system of $(a,-a$, $\left.c,-c ; x_{0}, y_{0}\right)$-additive mappings.

Proof. Put $u=a x-a x^{\prime}+x_{0}$ and $u^{\prime}=x^{\prime}$ for each $x, x^{\prime} \in X$. Then, $(\dagger)$ changes into

$$
\begin{aligned}
& \left\|f\left(a^{-1} u+u^{\prime}-a^{-1} x_{0}\right)-c^{-1} f(u)-f\left(u^{\prime}\right)+c^{-1} y_{0}\right\| \\
& \quad \leq \varepsilon_{1}\left(u, u^{\prime}\right),
\end{aligned}
$$

where

$$
\varepsilon_{1}\left(u, u^{\prime}\right)=\frac{1}{|c|} \varepsilon\left(a^{-1} u+u^{\prime}-a^{-1} x_{0}, u^{\prime}\right)
$$

for all $u, u^{\prime} \in X$. By denoting $a_{1}=a^{-1}, b_{1}=1, c_{1}=c^{-1}$, $d_{1}=1, u_{0}=-a^{-1} x_{0}$ and $v_{0}=-c^{-1} y_{0}$ in (19), ( $\dagger$ ) changes up to the following estimate of $f$ by the control function $\varepsilon_{1}$ :

$$
\left\|f\left(a_{1} u+b_{1} u^{\prime}+u_{0}\right)-c_{1} f(u)-d_{1} f\left(u^{\prime}\right)-v_{0}\right\| \leq \varepsilon_{1}\left(u, u^{\prime}\right)
$$

for all $u, u^{\prime} \in X$.

Under these transformations, $a_{1}, b_{1}, c_{1}, d_{1}$, and $\varepsilon_{1}$ are equipped with

$$
\begin{gathered}
a_{1}+b_{1} \neq 0, \quad K\left|c_{1}+d_{1}\right|<1, \\
\varepsilon_{1}\left(u, u^{\prime}\right) \leq K \varepsilon_{1}\left(\left(a_{1}+b_{1}\right) u+u_{0},\left(a_{1}+b_{1}\right) u^{\prime}+u_{0}\right)
\end{gathered}
$$

for all $u, u^{\prime} \in X$. The latter follows from the inequality in which $\varepsilon$ must satisfy because by using (20), we get

$$
\begin{gathered}
|c| \varepsilon_{1}\left(u, u^{\prime}\right)=\varepsilon\left(a^{-1} u+u^{\prime}+u_{0}, u^{\prime}\right)=\varepsilon\left(x, x^{\prime}\right), \\
\varepsilon\left(\left(a^{-1}+1\right) x-a^{-1} x_{0},\left(a^{-1}+1\right) x^{\prime}-a^{-1} x_{0}\right) \\
=\varepsilon\left(\left(a^{-1}+1\right) x-a^{-1} x_{0},\left(a_{1}+b_{1}\right) u^{\prime}+u_{0}\right) \\
=\varepsilon\left(\left(a^{-1}+1\right)\left(a^{-1} u+u^{\prime}+u_{0}\right)\right. \\
\left.\quad-a^{-1} x_{0},\left(a_{1}+b_{1}\right) u^{\prime}+u_{0}\right) \\
=\varepsilon\left(a^{-1}\left(\left(a_{1}+b_{1}\right) u+u_{0}\right)\right. \\
\quad+\left(\left(a_{1}+b_{1}\right) u^{\prime}+u_{0}\right) \\
\left.\quad-a^{-1} x_{0},\left(a_{1}+b_{1}\right) u^{\prime}+u_{0}\right) \\
=|c| \varepsilon_{1}\left(\left(a_{1}+b_{1}\right) u+u_{0},\left(a_{1}+b_{1}\right) u^{\prime}+u_{0}\right)
\end{gathered}
$$

for all $u, u^{\prime} \in X$. Since (21) and (22) hold, it follows from Theorem $\mathrm{A}$ that there exists a unique $\left(a_{1}, b_{1}, c_{1}, d_{1} ; u_{0}, v_{0}\right)$ additive mapping $f_{\infty}$ such that

$$
\left\|f(x)-f_{\infty}(x)\right\| \leq \frac{K \varepsilon_{1}(x, x)}{1-K\left|c_{1}+d_{1}\right|}
$$


for all $x \in X$. However, we can easily see the following two assertions:

(i) $f_{\infty}$ is $\left(a_{1}, b_{1}, c_{1}, d_{1} ; u_{0}, v_{0}\right)$-additive if and only if $f_{\infty}$ is $\left(a,-a, c,-c ; x_{0}, y_{0}\right)$-additive,

(ii) (24) is equivalent to ( $)$.

This completes the proof.

Theorem 8. Let $c+1 \neq 0$ and $K \geq 0$ with $K|c|<|c+1|$. One takes a control function $\varepsilon$ which satisfies

$$
\begin{aligned}
& \varepsilon\left(\left(a^{-1}+1\right) x-a^{-1} x_{0},\left(a^{-1}+1\right) x^{\prime}-a^{-1} x_{0}\right) \\
& \leq K \varepsilon\left(x, x^{\prime}\right)
\end{aligned}
$$

for all $x, x^{\prime} \in X$ and puts

$$
\delta(x)=\frac{\varepsilon\left(\left(a^{-1}+1\right) x-a^{-1} x_{0}, x\right)}{|c+1|-K|c|}
$$

for each $x \in X$.

Then the strict $(\varepsilon, \delta)$-stability holds for the system of $(a,-a$, $\left.c,-c ; x_{0}, y_{0}\right)$-additive mappings.

Proof. We consider the same transformations and the same estimate (21) of $f$ by $\varepsilon_{1}$ in the proof of Theorem 7 . Under these transformations, we have

$$
c_{1}+d_{1} \neq 0, \quad K<\left|c_{1}+d_{1}\right| .
$$

Moreover, for every $u, u^{\prime} \in X$ we have

$$
\varepsilon_{1}\left(\left(a_{1}+b_{1}\right) u+u_{0},\left(a_{1}+b_{1}\right) u^{\prime}+u_{0}\right) \leq K \varepsilon_{1}\left(u, u^{\prime}\right)
$$

because

$$
\begin{aligned}
& |c| \varepsilon_{1}\left(u, u^{\prime}\right)=\varepsilon\left(a^{-1} u+u^{\prime}+u_{0}, u^{\prime}\right)=\varepsilon\left(x, x^{\prime}\right), \\
& |c| \varepsilon_{1}\left(\left(a_{1}+b_{1}\right) u+u_{0},\left(a_{1}+b_{1}\right) u^{\prime}+u_{0}\right) \\
& \quad=\varepsilon\left(\left(a^{-1}+1\right) x-a^{-1} x_{0},\left(a^{-1}+1\right) x^{\prime}-a^{-1} x_{0}\right) .
\end{aligned}
$$

Since (21), (27) and (28) hold, it follows from Theorem B that there exists a unique $\left(a_{1}, b_{1}, c_{1}, d_{1} ; u_{0}, v_{0}\right)$-additive mapping $f_{\infty}$ such that

$$
\left\|f(x)-f_{\infty}(x)\right\| \leq \frac{\varepsilon_{1}(x, x)}{\left|c_{1}+d_{1}\right|-K}
$$

for all $x \in X$. This means $(\ddagger)$ and $f_{\infty}$ is $\left(a,-a, c,-c ; x_{0}, y_{0}\right)$ additive.

Theorem 9. Let $0 \leq K<1 / 2$. One takes a control function $\varepsilon$ which satisfies

$$
\varepsilon\left(x, x^{\prime}\right) \leq K \varepsilon\left(2 x-x_{0}, 2 x^{\prime}-x_{0}\right)
$$

for all $x, x^{\prime} \in X$ and puts

$$
\delta(x)=\frac{K \varepsilon\left(x, 2 x-x_{0}\right)}{1-2 K}
$$

for each $x \in X$.

Then the strict $(\varepsilon, \delta)$-stability holds for the system of $\left(-1,1,-1,1 ; x_{0}, y_{0}\right)$-additive mappings.

Proof. Put $u=x, u^{\prime}=-x+x^{\prime}+x_{0}$ for each $x, x^{\prime} \in X$. Then, $(\dagger)$ changes into the following estimate of $f$ by the control function $\varepsilon_{1}$ :

$$
\left\|f\left(u+u^{\prime}-x_{0}\right)-f(u)-f\left(u^{\prime}\right)+y_{0}\right\| \leq \varepsilon_{1}\left(u, u^{\prime}\right),
$$

where

$$
\varepsilon_{1}\left(u, u^{\prime}\right)=\varepsilon\left(u, u+u^{\prime}-x_{0}\right) .
$$

for all $u, u^{\prime} \in X$. Put $a_{1}=b_{1}=c_{1}=d_{1}=1$.

Under these transformations, $a_{1}, b_{1}, c_{1}, d_{1}$, and $\varepsilon_{1}$ are equipped with

$$
\begin{gathered}
a_{1}+b_{1}=2 \neq 0, \quad K\left|c_{1}+d_{1}\right|=2 K<1, \\
\varepsilon_{1}\left(u, u^{\prime}\right) \leq K \varepsilon_{1}\left(\left(a_{1}+b_{1}\right) u-x_{0},\left(a_{1}+b_{1}\right) u^{\prime}-x_{0}\right)
\end{gathered}
$$

for all $u, u^{\prime} \in X$. The latter follows from the following inequality:

$$
\begin{aligned}
\varepsilon_{1}\left(u, u^{\prime}\right) & =\varepsilon\left(u, u+u^{\prime}-x_{0}\right) \\
& \leq K \varepsilon\left(2 u-x_{0}, 2\left(u+u^{\prime}-x_{0}\right)-x_{0}\right) \\
& =K \varepsilon\left(2 u-x_{0},\left(2 u-x_{0}\right)+\left(2 u^{\prime}-x_{0}\right)-x_{0}\right) \\
& =K \varepsilon_{1}\left(2 u-x_{0}, 2 u^{\prime}-x_{0}\right)
\end{aligned}
$$

for all $u, u^{\prime} \in X$. Since (34), (36) hold, it follows from Theorem $\mathrm{A}$ that there exists a unique $\left(a_{1}, b_{1}, c_{1}, d_{1} ;-x_{0},-y_{0}\right)$ additive mapping $f_{\infty}$ such that

$$
\left\|f(x)-f_{\infty}(x)\right\| \leq \frac{K \varepsilon_{1}(x, x)}{1-K\left|c_{1}+d_{1}\right|}
$$

for all $x \in X$. However we can easily see the following two assertions:

(i) $f_{\infty}$ is $\left(a_{1}, b_{1}, c_{1}, d_{1} ;-x_{0},-y_{0}\right)$-additive if and only if $f_{\infty}$ is $\left(-1,1,-1,1 ; x_{0}, y_{0}\right)$-additive;

(ii) (38) is equivalent to ( $¥$ ).

This completes the proof.

Theorem 10. Let $0 \leq K<2$. One takes a control function $\varepsilon$ which satisfies

$$
\varepsilon\left(2 x-x_{0}, 2 x^{\prime}-x_{0}\right) \leq K \varepsilon\left(x, x^{\prime}\right)
$$


for all $x, x^{\prime} \in X$ and puts

$$
\delta(x)=\frac{\varepsilon\left(x, 2 x-x_{0}\right)}{2-K}
$$

for each $x \in X$.

Then the strict $(\varepsilon, \delta)$-stability holds for the system of $\left(-1,1,-1,1 ; x_{0}, y_{0}\right)$-additive mappings.

Proof. We consider the same transformation and the same estimate (34) of $f$ by $\varepsilon_{1}$ in the proof of Theorem 9. Under these transformations, $c_{1}, d_{1}$ and $\varepsilon_{1}$ are equipped with

$$
\begin{aligned}
& c_{1}+d_{1}=2 \neq 0, \quad K<2=\left|c_{1}+d_{1}\right|, \\
\varepsilon_{1}\left(2 u-x_{0}, 2 u^{\prime}-x_{0}\right) & \\
= & \varepsilon\left(2 u-x_{0},\left(2 u-x_{0}\right)+\left(2 u^{\prime}-x_{0}\right)-x_{0}\right) \\
& =\varepsilon\left(2 x-x_{0}, 2\left(u+u^{\prime}-x_{0}\right)-x_{0}\right) \\
& =\varepsilon\left(2 x-x_{0}, 2 x^{\prime}-x_{0}\right) \\
\leq & K \varepsilon\left(x, x^{\prime}\right) \\
= & K \varepsilon\left(u, u+u^{\prime}-x_{0}\right) \\
= & K \varepsilon_{1}\left(u, u^{\prime}\right)
\end{aligned}
$$

for all $u, u^{\prime} \in X$. So it follows that

$$
\varepsilon_{1}\left(\left(a_{1}+b_{1}\right) u-x_{0},\left(a_{1}+b_{1}\right) u^{\prime}-x_{0}\right) \leq K \varepsilon_{1}\left(u, u^{\prime}\right)
$$

for all $u, u^{\prime} \in X$. Since (34), (41) and (43) hold, it follows from Theorem $\mathrm{B}$ that there exists a unique $\left(a_{1}, b_{1}, c_{1}, d_{1} ;-x_{0},-y_{0}\right)$ additive mapping $f_{\infty}$ such that

$$
\left\|f(x)-f_{\infty}(x)\right\| \leq \frac{\varepsilon_{1}(x, x)}{\left|c_{1}+d_{1}\right|-K}
$$

for all $x, x^{\prime} \in X$. This means ( $\left.\ddagger\right)$ and $f_{\infty}$ is $(-1,1,-1$, $\left.1 ; x_{0}, y_{0}\right)$-additive.

\section{Concrete Examples}

Throughout this section, let $x_{0}=y_{0}=0$. We fix nonnegative constants $p, q$ and $\delta$ and take the control function $\varepsilon$ defined by $\varepsilon\left(x, x^{\prime}\right)=\delta\|x\|^{p}\left\|x^{\prime}\right\|^{q}$ for every $x, x^{\prime} \in X$.

Corollary 11. When $a+1 \neq 0$ and $|a|^{p+q}|c+1|<|c||a+1|^{p+q}$, one puts

$$
\delta^{\prime}(x)=\frac{\delta|a+1|^{p}|a|^{q}\|x\|^{p+q}}{|c||a+1|^{p+q}-|c+1||a|^{p+q}}
$$

for each $x \in X$.

Then, the system of $(a,-a, c,-c)$-additive mappings is strictly $\left(\varepsilon, \delta^{\prime}\right)$-stable.
Proof. Put $K=\left|a^{-1}+1\right|^{-(p+q)}$. Then $K|c+1|<|c|$ and

$$
\varepsilon\left(x, x^{\prime}\right)=K \varepsilon\left(\left(a^{-1}+1\right) x,\left(a^{-1}+1\right) x^{\prime}\right)
$$

for all $x, x^{\prime} \in X$. By Theorem 7, for a mapping $f$ of $X$ into $Y$ satisfying ( $\dagger$ ) for $a=-b$ and $c=-d$, there exists a unique $(a,-a, c,-c)$-additive mapping $f_{\infty}$ such that

$$
\left\|f(x)-f_{\infty}(x)\right\| \leq \frac{K \delta\left|a^{-1}+1\right|^{p}\|x\|^{p+q}}{|c|-K|c+1|}
$$

for all $x, x^{\prime} \in X$. Because of $K=\left|a^{-1}+1\right|^{-(p+q)}$, we have the corollary.

Corollary 12. When $c+1 \neq 0$ and $|a+1|^{p+q}|c|<|c+1||a|^{p+q}$, one puts

$$
\delta^{\prime}(x)=\frac{\delta|a+1|^{p}|a|^{q}\|x\|^{p+q}}{|a|^{p+q}|c+1|-|a+1|^{p+q}|c|}
$$

for each $x \in X$.

Then the system of $(a,-a, c,-c)$-additive mappings is strictly $\left(\varepsilon, \delta^{\prime}\right)$-stable.

Proof. Put $K=\left|a^{-1}+1\right|^{p+q}$. Then $K|c|<|c+1|$ and

$$
\varepsilon\left(\left(a^{-1}+1\right) x,\left(a^{-1}+1\right) x^{\prime}\right)=K \varepsilon\left(x, x^{\prime}\right)
$$

for all $x, x^{\prime} \in X$. By Theorem 8 , for a mapping $f$ of $X$ into $Y$ satisfying ( $\dagger$ ) for $a=-b$ and $c=-d$, there exists a unique $(a,-a, c,-c)$-additive mapping $f_{\infty}$ such that

$$
\left\|f(x)-f_{\infty}(x)\right\| \leq \frac{\delta\left|a^{-1}+1\right|^{p}\|x\|^{p+q}}{|c+1|-K|c|}
$$

for all $x \in X$. Because of $K=\left|a^{-1}+1\right|^{p+q}$, we have the corollary.

Corollary 13. When $p+q>1$, one puts

$$
\delta^{\prime}(x)=\frac{2^{q} \delta\|x\|^{p+q}}{2^{p+q}-2}
$$

for each $x \in X$.

Then, the system of $(-1,1,-1,1)$-additive mappings is $\operatorname{strictly}\left(\varepsilon, \delta^{\prime}\right)$-stable.

Proof. Put $K=2^{-(p+q)}$. Then,

$$
\varepsilon\left(x, x^{\prime}\right)=K \varepsilon\left(2 x, 2 x^{\prime}\right)
$$

for all $x, x^{\prime} \in X$. Since $p+q>1$, we also have $0 \leq K<1 / 2$. By Theorem 9, for a mapping $f$ of $X$ into $Y$ satisfying $(\dagger)$ for $a=c=-1$ and $b=d=1$, there exists a unique $(-1,1,-1,1)$ additive mapping $f_{\infty}$ such that

$$
\left\|f(x)-f_{\infty}(x)\right\| \leq \frac{2^{q} \delta K\|x\|^{p+q}}{1-2 K}
$$

for all $x \in X$. Because of $K=2^{-(p+q)}$, we have the corollary. 
Corollary 14. When $p+q<1$, one puts

$$
\delta^{\prime}(x)=\frac{2^{q} \delta\|x\|^{p+q}}{2-2^{p+q}}
$$

for each $x \in X$.

Then, the system of $(-1,1,-1,1)$-additive mappings is $\operatorname{strictly}\left(\varepsilon, \delta^{\prime}\right)$-stable.

Proof. Put $K=2^{p+q}$. Then,

$$
\varepsilon\left(2 x, 2 x^{\prime}\right)=K \varepsilon\left(x, x^{\prime}\right)
$$

for all $x, x^{\prime} \in X$. Since $p+q<1$, we also have $0 \leq K<2$. By Theorem 10, for a mapping $f$ of $X$ into $Y$ satisfying $(\dagger)$ for $a=c=-1$ and $b=d=1$, there exists a unique $(-1,1,-1,1)$ additive mapping $f_{\infty}$ such that

$$
\left\|f(x)-f_{\infty}(x)\right\| \leq \frac{2^{q} \delta\|x\|^{p+q}}{2-K}
$$

for all $x \in X$. Because of $K=2^{p+q}$, we have the corollary.

Remark 15. In Corollary 14, taking $p=q=0$, we can easily observe that the corollary is just the stability result due to Hyers [2, Theorem 1].

\section{Acknowledgment}

The third and fifth authors are partially supported by Grantin-Aid for Scientific Research, Japan Society for the Promotion of Science.

\section{References}

[1] S. M. Ulam, A Collection of Mathematical Problems, vol. 8 of Interscience Tracts in Pure and Applied Mathematics, Interscience Publishers, New York, NY, USA, 1960.

[2] D. H. Hyers, "On the stability of the linear functional equation," Proceedings of the National Academy of Sciences of the United States of America, vol. 27, pp. 222-224, 1941.

[3] T. Aoki, "On the stability of the linear transformation in Banach spaces," Journal of the Mathematical Society of Japan, vol. 2, pp. 64-66, 1950.

[4] T. M. Rassias, "On the stability of the linear mapping in Banach spaces," Proceedings of the American Mathematical Society, vol. 72, no. 2, pp. 297-300, 1978.

[5] J. Aczél, Lectures on Functional Equations and Their Applications, vol. 19 of Mathematics in Science and Engineering, Academic Press, New York, NY, USA, 1966.

[6] S.-E. Takahasi, T. Miura, and H. Takagi, "On a Hyers-UlamAoki-Rassias type stability and a fixed point theorem," Journal of Nonlinear and Convex Analysis, vol. 11, no. 3, pp. 423-439, 2010.

[7] S. -E. Takahasi, T. Miura, H. Takagi, and K. Tanahashi, "Ulam type stability problems for alternative homomorphisms".

[8] M. Todoroki, K. Kumahara, T. Miura, and S.-E. Takahasi, "Stability problems for generalized additive mappings and Euler-Lagrange type mappings," The Australian Journal of Mathematical Analysis and Applications, vol. 9, no. 1, article 19, 2012. 


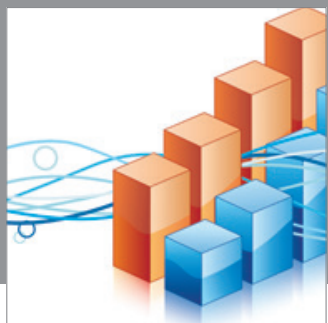

Advances in

Operations Research

mansans

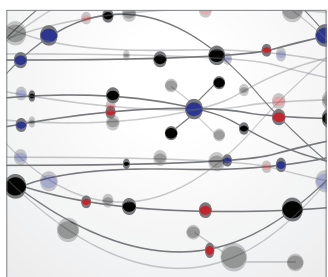

The Scientific World Journal
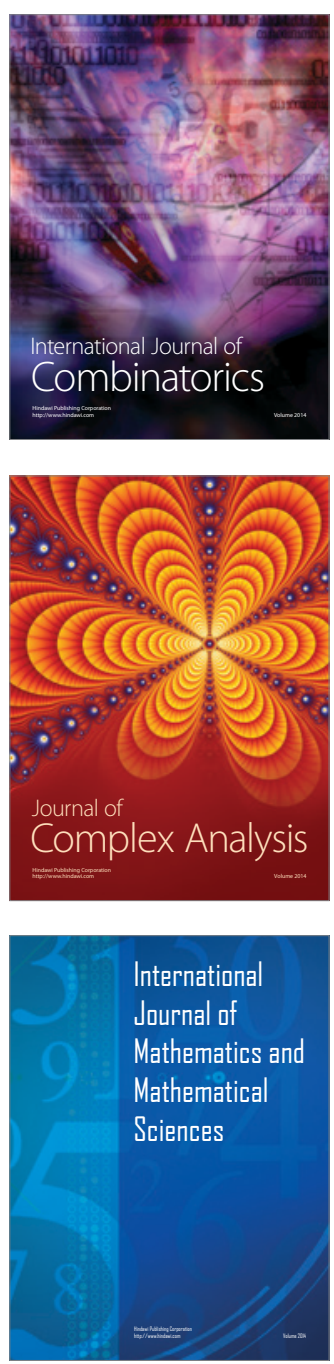
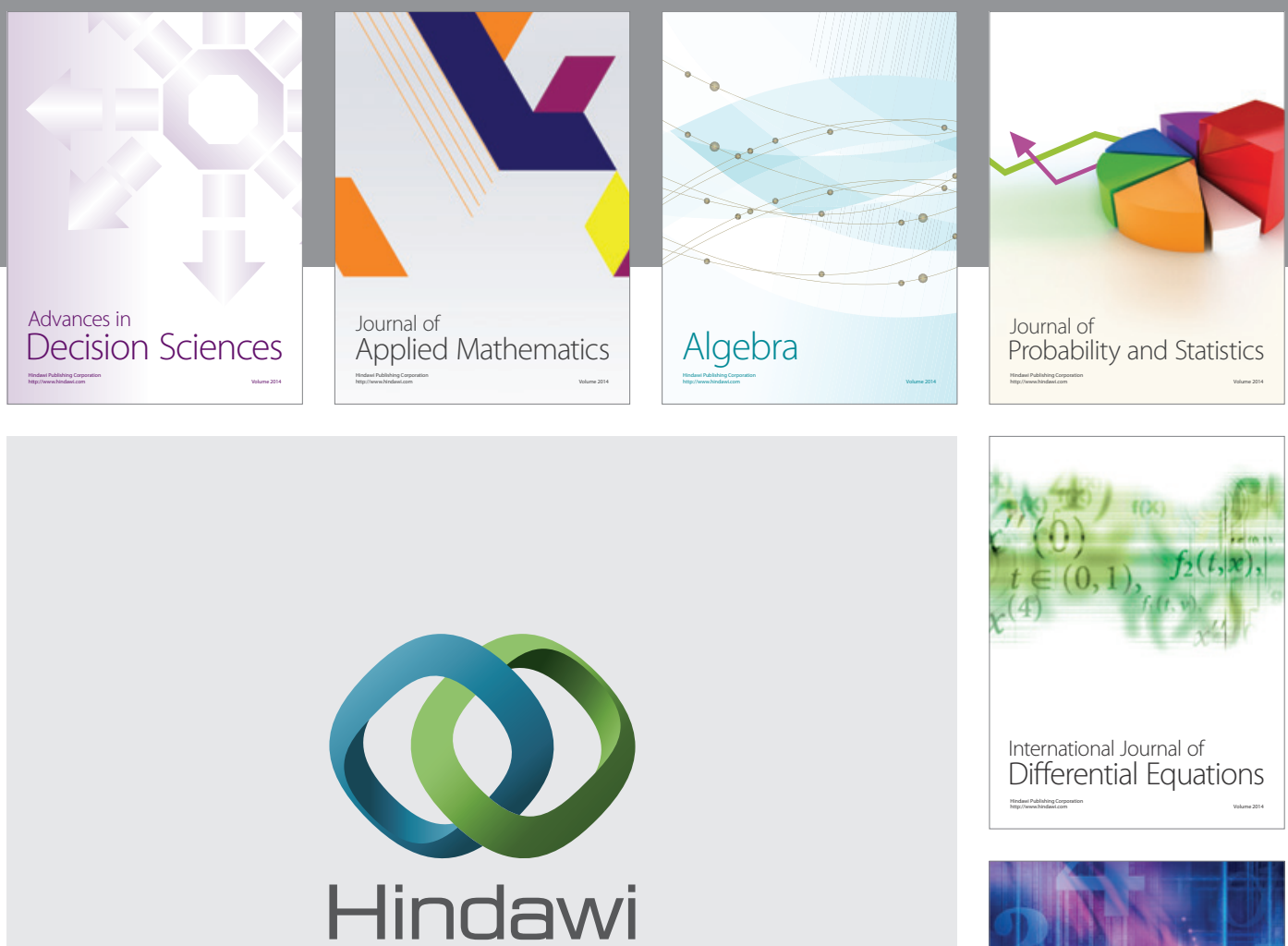

Submit your manuscripts at http://www.hindawi.com
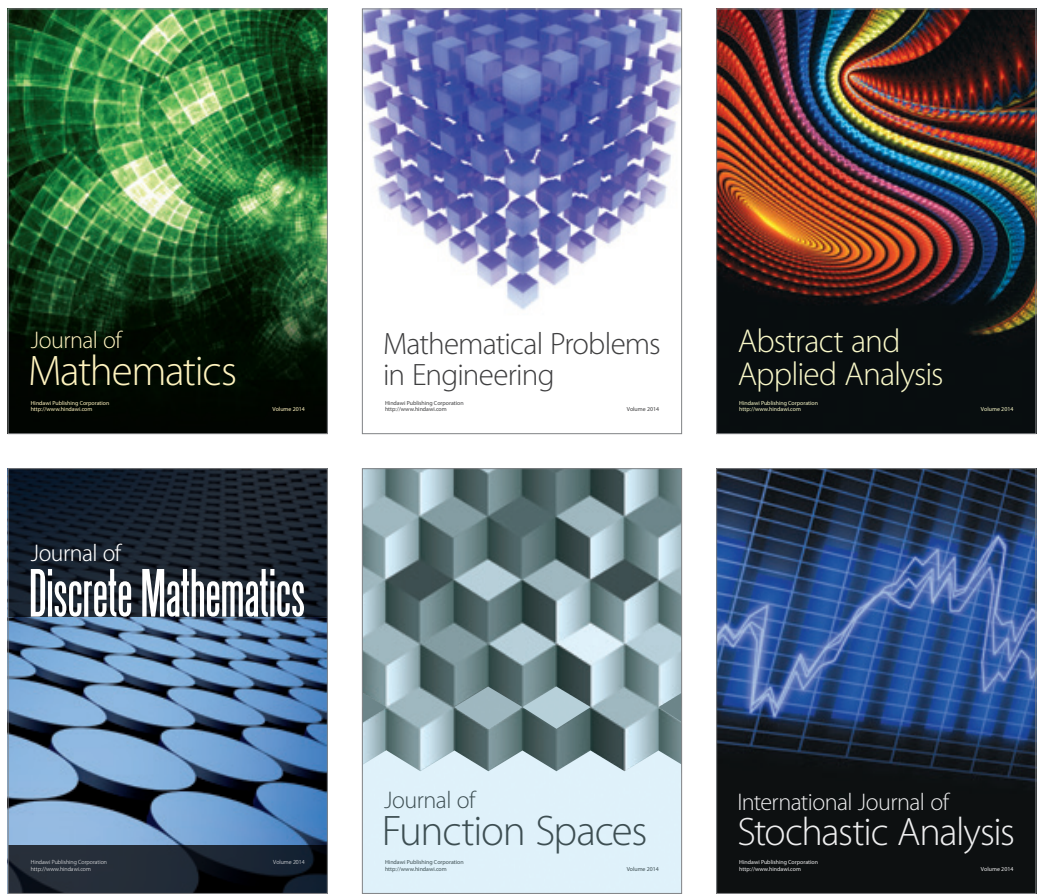

Journal of

Function Spaces

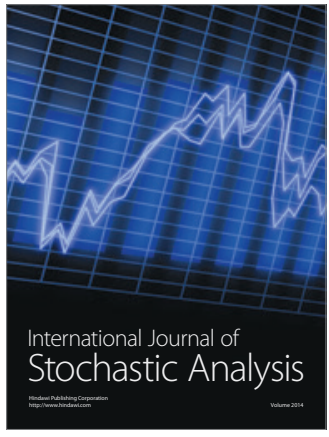

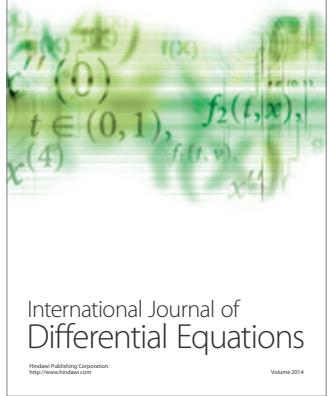
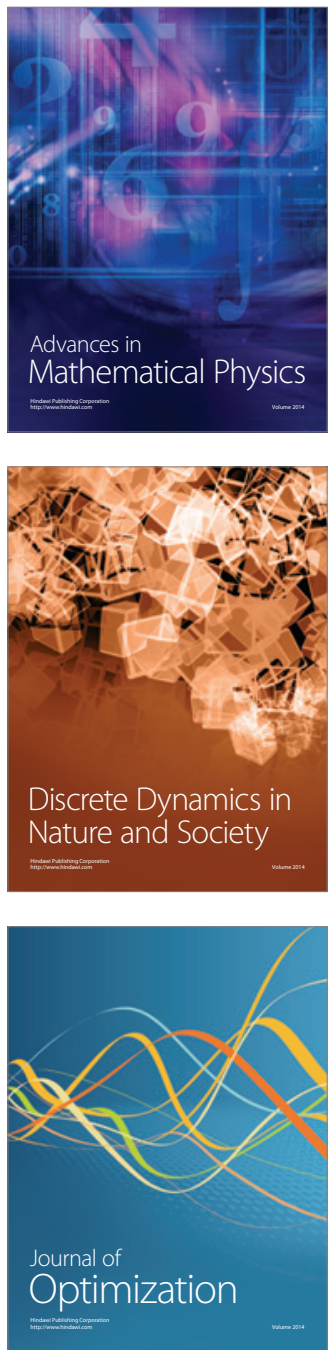